\title{
Hsa_circ_0002060 Knockdown Ameliorates Osteoporosis by Targeting MiR-198-5p
}

\author{
Man Liu, ${ }^{a}$ Yao Meng, ${ }^{b}$ Keren He, ${ }^{*, a}$ and Chonglin Luan ${ }^{c}$ \\ ${ }^{a}$ School of Medical Technology and Nursing, Shenzhen Polytechnic; Shenzhen, Guangdong 508055, China: \\ ${ }^{b}$ Orthodontic Center, Shenzhen Children's Hospital; Shenzhen, Guangdong 518026, China: and ${ }^{c}$ Chemical College, \\ Shenzhen Polytechnic; Shenzhen, Guangdong 508055, China. \\ Received August 13, 2020; accepted October 23, 2020; advance publication released online November 5, 2020
}

\begin{abstract}
Osteoporosis (OP) is increasingly becoming one of a major health concerns all over the world. However, the limitations of current therapeutic drugs for OP are including considerable side effects and low efficacy. Therefore, it is required to develop new therapeutic drugs for OP. This study aimed to investigate the role of hsa_circ_0002060 in the regulation of osteoporosis. Osteoblast cells (hFOB 1.19) were transfected with hsa_circ_0002060 small interfering RNA (siRNA), following by stimulated with dexamethasone (DEX) to mimic OP in vitro. Cell counting kit-8, apoptosis, and JC-1 mitochondrial membrane potential assays were used to evaluate the cell viability, apoptosis, and mitochondrial membrane potential, respectively. Western blot was conducted to detect the expression of proteins. In addition, the levels of reactive oxygen species, superoxide dismutase, glutathione and malondialdehyde were measured with enzyme-linked immunosorbent assay (ELISA). The putative target of hsa_circ_0002060 was verified by dual luciferase reporter assay and RNA pull down. At last, the role of hsa_circ_0002060 in the progression of OP was investigated with an ovariectomy (OVX)-induced OP mouse model. The results indicated DEX could induce cell viability decline in hFOB 1.19 cells, which was ameliorated by hsa_circ_0002060 knockdown. Consistently, DEX-induced cell apoptosis of hFOB 1.19 was ameliorated by hsa_circ_0002060 knockdown as well. As for the underlying mechanisms study, hsa_circ_0002060 was proved to regulate the viability of hFOB 1.19 cells through targeting miR-198-5p/Bax axis. Additionally, hsa_circ_0002060 knockdown alleviated ovariectomy-induced OP in a mouse model. Taken together, hsa_circ_0002060 knockdown alleviated the progression of OP by targeting miR-198-5p. Hsa_circ_0002060 might possibly be served as a therapeutic target for treating OP.
\end{abstract}

Key words osteoporosis; hsa_circ_0002060; miR-198-5p

\section{INTRODUCTION}

Osteoporosis (OP) is a common type of chronic systemic skeletal diseases which is characterized by low bone mass and microarchitectural deterioration of bone tissue. ${ }^{1)}$ The consequences of OP include increased bone fragility and augmented susceptibility to bone fracture. ${ }^{1)}$ Eventually, OP will lead to pain, severe disability and premature death from fracture. ${ }^{1)}$ As aging population is growing, OP is increasingly becoming one of a major health concerns all over the world. ${ }^{1)}$ At present, more than 200 million people are effected by OP all over the world. ${ }^{2)}$ Moreover, 1 in 3 women ( $>50$ years old) and 1 in 5 men will experience osteoporotic fractures in their lifetime worldwide. ${ }^{2)}$ Therapeutic drugs for OP include anti-resorptive agents, anabolic medications and some new drugs such as tissue-selective estrogen receptor complexes (TSECs). ${ }^{1)}$ However, the limitations of current therapeutic drugs for OP are including considerable side effects and low efficacy. ${ }^{1)}$ Ideal therapeutic drugs have not yet been developed yet. ${ }^{1)}$ Therefore, it is required to develop new therapeutic drugs for OP.

Circular RNAs (circRNAs) are novel clusters of noncoding RNAs which are emerging as important regulators of gene expression. ${ }^{3,4)}$ CircRNAs regulate target gene expression through interacting with microRNAs (miRNAs) as competitive endogenous RNAs (ceRNAs). ${ }^{5}$ Previous studies revealed that circRNAs acted as important regulators in human diseases. ${ }^{3)}$ A study of Huang et al. demonstrated that hsa_circ_0002060 was significantly upregulated in patients with OP and associ- ated with the low bone mineral density in these patients. However, the role of hsa_circ_0002060 in OP remains unclear. This study aimed to investigate the role of hsa_circ_0002060 in the regulation of cell viability of osteoblast.

\section{MATERIALS AND METHODS}

Reagents Dexamethasone (DEX) was purchased from Sigma-Aldrich (St. Louis, MO, U.S.A.). All antibodies were obtained from Abcam (Cambridge, MA, U.S.A.).

Cell Culture The hFOB1.19 cells were obtained from American Type Culture Collection (Manassas, VA, U.S.A.) and cultured in Dulbecco's modified Eagle's medium (DMEM)/F12 medium (GIBCO, Grand Island, NY, U.S.A.) supplemented with $10 \%$ fetal bovine serum (GIBCO), $2.5 \mathrm{mM} / \mathrm{L}$ glutamine (Sigma-Aldrich) and $0.3 \mathrm{mg} / \mathrm{mL}$ G418 (Sigma-Aldrich). The cells were cultured at $37^{\circ} \mathrm{C}$ in a humidified atmosphere of $5 \% \mathrm{CO}_{2}$.

Knockdown of Hsa_circ_0002060 Hsa_circ_0002060 siRNAs and negative control (NC) were designed and synthesized by GenePharma (Shanghai, China). When hFOB 1.19 cells reached $80 \%$ confluence in 6 -well plates, the cells were transfected with small interfering RNA (siRNA) or NC using Lipofectamine 2000 (Invitrogen, Carlsbad, CA, U.S.A.) for $48 \mathrm{~h}$.

Cell Viability Assay The hFOB1.19 cells were transfected with siRNA of hsa_circ_0002060 for $48 \mathrm{~h}$, following treated with DEX for $24 \mathrm{~h}$. After indicated treatment, cell viability 
assay was performed using the cell counting kit-8 (CCK-8, Beyotime, Jiangsu, China) according to the manufacturer's instructions. Briefly, the cells were incubated with $10 \mu \mathrm{L} \mathrm{CCK}-8$ reagent for $3 \mathrm{~h}$ at $37^{\circ} \mathrm{C}$. Absorbance was measured at $450 \mathrm{~nm}$ using a spectrophotometer.

Ki67 Staining Cell proliferation was detected by immunofluorescence staining for Ki67 as well. Briefly, the cells were fixed with paraformaldehyde (4\%) for $15 \mathrm{~min}$ and treated with $0.1 \%$ Triton $\mathrm{X}-100$ for $10 \mathrm{~min}$ at room temperature. Then, the cells were incubated with primary antibodies for Ki67 (1: 500, Abcam) overnight at $4{ }^{\circ} \mathrm{C}$. After that, cells were incubated with mouse anti-rabbit secondary antibody $(1: 1000$, Abcam) at $37^{\circ} \mathrm{C}$ for $1 \mathrm{~h}$. Cell nucleus were counter stained with $5 \mu \mathrm{g} / \mathrm{mL}$ 4,6-diamidino-2-phenyl-indole (DAPI) for $30 \mathrm{~min}$ at room temperature.

Real-Time Quantitative RT-PCR (RT-qPCR) Total RNAs were isolated from hFOB1.19 cells with TRIzol reagent (TaKaRa Biotechnology, Dalian, China). Then, the isolated RNAs were reversely transcribed into cDNAs with a PrimeScript RT reagent kit following the manufacturer's procedures (TaKaRa). RT-qPCR reactions were carried out on an applied biosystems (ABI) 7900 system (Applied Biosystems, Foster City, CA, U.S.A.) using SYBR $^{\circledR}$ Premix Ex Taq ${ }^{\mathrm{TM}}$ II kit (TaKaRa). Relative gene expression was calculated using $2^{-\triangle \triangle \mathrm{Ct}}$ method. The results were normalized to the level of ACTIN or U6 expression. The sequences of primers were listed as below: hsa_circ_0002060, forward, 5'-GCC ACA GGTCTA AAA CAGGCT T-3', reverse, $\quad 3$ '-CGC AGT GCC CTC TGA ATA GG-5'; miR-198-5p, forward, 5'-GGT CCA GAGGGG AGA TAG GT-3', reverse, 3'-CTC AAC TGG TGT CGT GGA GTC-5'; U6, forward, 5'-CTC GCT TCG GCA GCA CAT-3', reverse, 5'-AAC GCT TCA CGAATT TGC GT-3'; ACTIN, forward, 5'-GTC CAC CGC AAA TGC TTC TA-3', reverse, 3'-TGC TGT CAC CTT CAC CGT TC-5'.

Detection of Cell Apoptosis The detection and quantification of apoptotic cells were performed using Annexin V-fluorescein isothiocyanate (FITC) Apoptosis Detection Kit (Thermo Fisher Scientific, Waltham, MA, U.S.A.) and flow cytometry. Briefly, the cells were seeded into 6-well plates and cultured overnight. Then, the cells were transfected with the siRNA for $48 \mathrm{~h}$, following treated with DEX for $24 \mathrm{~h}$. Then, the cultured cells were harvested, washed with cold phosphate buffered saline (PBS) for three times, and stained with Annexin $\mathrm{V}$ and propidium iodide (PI) for $15 \mathrm{~min}$ at room temperature in the dark following the manufacture's protocol. The cell apoptosis was detected and analyzed on a FACScan flow cytometer using Cell Quest software (Becton Dickinson, San Jose, CA, U.S.A.).

Western Blot Analysis Total proteins were extracted using radio immunoprecipitation assay (RIPA) buffer (Beyotime). The concentration of protein was measured using bicinchoninic acid (BCA) Protein Assay Kit (Beyotime). Equal amounts of proteins $(40 \mu \mathrm{g})$ were subjected to electrophoresis on $10 \%$ sodium dodecyl sulfate-polyacrylamide gel (SDS-PAGE) and transferred onto a polyvinylidene difluoride (PVDF) membrane. After blocking with 5\% skim milk, the membranes were incubated with primary antibodies overnight at $4{ }^{\circ} \mathrm{C}$. Then, the membranes were incubated with goat antirabbit secondary antibodies $(1: 2000)$ for $2 \mathrm{~h}$ at room temperature. The blots were visualized using chemiluminescence substrate (Thermo Fisher Scientific). The used primary antibodies were anti-Bax $(1: 1000)$, anti-bcl-2 $(1: 1000)$, anti-cleaved caspase $9(1: 1000)$, anti-cleaved caspase 3 (1:1000), anti-p-c-Jun N-terminal kinase (JNK) $(1: 1000)$ and anti-JNK $(1: 1000)$.

Cell Transfection MiR-198-5p mimics, miR-198-5p inhibitor and negative control (NC) were synthesized by GenePharma. The hFOB1.19 cells were transfected with miR-198-5p mimics, miR-198-5p inhibitor or NC using Lipofectamine 2000 (Invitrogen) for $48 \mathrm{~h}$ according to the manufacturer's procedures. The efficacy of transfection was verified by RTqPCR.

Luciferase Reporter Assay The target miRNA of hsa_circ_0002060 was predicted by CircInteractome online database (https://circinteractome.nia.nih.gov/index.html). MiR-198-5p was predicted as the target miRNA of hsa_circ_0002060. Then, the downstream target of miR-198-5p was predicted using TargetScan (www.targetscan.org/vert_71) and miRDB (http://www.mirdb.org/) online database. For Luciferase reporter assay, wild type (WT) or mutation type (MT) of the putative binding site of hsa_circ_0002060 was inserted into pmiR-RB-Report ${ }^{\mathrm{TM}}$ luciferase vector (Promega Corporation, Madison, WI, U.S.A.). The hFOB1.19 cells were cotransfected with WT or MT reporter vectors and miR-198-5p mimics or vector control (vector-ctrl) using Lipofectamine 2000 (Invitrogen). After $48 \mathrm{~h}$ of transfection, luciferase activities were detected using the dual-luciferase reporter gene assay kit (Promega) as per the manufacturer's protocols. To verify the relationship between miR-198-5p and Bax, WT or MT of the putative binding site of Bax was inserted into pmiR-RB-Report ${ }^{\mathrm{TM}}$ luciferase vector (Promega). The following steps were same as descripted above.

RNA Pull Down A Pierce ${ }^{\mathrm{TM}}$ Magnetic RNA-Protein Pull-Down Kit (Thermo Fisher Scientific) was used for RNA pulldown following the manufacturer's procedures. Hsa_circ_0002060 probe was prepared using T7 RiboMAX Express large-scale RNA production system (Promega). Then, hsa_circ_0002060 probe was biotin-labeled with a Pierce RNA 3'-end desthiobiotinylation kit (Thermo Fisher Scientific) following the manufacturer's instructions. Next, biotin-labeled hsa_circ_0002060 probe was bound with magnetic beads followed by binding with miRNAs from hFOB1.19 cell lysates. Retrieved miRNA was subjected to RT-qPCR.

Detection of Mitochondrial Membrane Potential (MMP) The hFOB1.19 cells cells $\left(2 \times 10^{5}\right.$ cells per well $)$ were seeded into 96-well plates and cultured overnight. Then, the cells were transfected with siRNA 2 with/without co-transfection with miR-198-5p inhibitor for $48 \mathrm{~h}$. Next, the cells were further incubated with $1 \mu \mathrm{M}$ DEX for $24 \mathrm{~h}$. MMP was examined using JC-1 kits (Beyotime). In brief, the cells from each group were incubated with $\mathrm{JC}-1$ reagent for $20 \mathrm{~min}$ at $37^{\circ} \mathrm{C}$ in the dark. After washing twice with JC-1 buffer solution, the fluorescence was observed using a fluorescence microscope.

Detection of Reactive Oxygen Species (ROS) After indicated treatment, the level of ROS in each group was estimated using 2',7'-dichlorodihydrofluorescein diacetate (DCFH-DA; Sigma-Aldrich) reagent as per the manufacturer's protocol. The fluorescent intensity of DCFH-DA was detected by flow cytometry.

Detection of Superoxide Dismutase (SOD), Glutathione (GSH), and Malondialdehyde (MDA) The activities of SOD, MDA, and GSH were detected using corresponding enzyme-linked immunosorbent assay (ELISA) kits (Nanjing 
Jiancheng Bioengineering Institute, Jiangsu, China) following the instructions of the manufacturer. Briefly, the cells from each group were harvested, washed, lysed and incubated with corresponding enzyme-specific substrates. Then, the absorbance at $450 \mathrm{~nm}$ was determined using a microplate reader (Bio-Rad Laboratories, Hercules, CA, U.S.A.).

Mice and Ovariectomy (OVX) Surgery All procedures involved animals were complied with the principles of the NIH Guide for the Care and Use of Laboratory Animals and were approved by the ethics committee for laboratory animal care and use of Shenzhen Polytechnic. Female C57BL/6 mice were provided by Vital River (Beijing, China). The mice were maintained under pathogen-free condition at $25^{\circ} \mathrm{C}$ on a $12-\mathrm{h}$ light/dark cycle with free access to food and water. At age of 16 weeks old, the mice were randomly divided into four groups ( $N=6$ per group): control, sham, bilaterally OVX and OVX + siRNA2. For OVX group, the mice were bilaterally ovariectomized. For sham group, the ovaries were exposed without removal. The mice were anesthetized with $1.5 \%$ isoflurane (Abbott Laboratories, IL, U.S.A.) in oxygen for surgery. The dorsal mid-lumbar area of each mouse was shaved. The shaved skin was cleaned with $70 \%$ ethanol. A midline dorsal skin incision $(2 \mathrm{~cm})$ was made and the pair of ovaries were excised. For SHAM group, the ovaries were exteriorized and then returned to the abdominal cavity of the mice. The mice of OVX + siRNA2 group were given siRNA2 $(50 \mathrm{nM})$ administration twice a week for 4 weeks by intravenous injection via lateral tail veins. The siRNA2 with chemical modifications for utilizing in vivo was synthesized by GenePharma (Shanghai, China).

Bone Mineral Density (BMD) and Hematoxylin-Eosin (H\&E) Staining Mice were sacrificed at the end of week 20.
Left femurs were harvested for bone mineral density analysis and $\mathrm{H} \& \mathrm{E}$ staining. BMD was determined using dual-energy X-ray absorptiometry scanner (DCS600EX-IIIR, ALOKA Co., Ltd., Tokyo, Japan). For H\&E staining, the femur was fixed in $10 \%$ formalin for $2 \mathrm{~d}$ and then embedded in paraffin. Sections were cut at $5 \mu \mathrm{m}$ thickness. The sections were stained with H\&E. The histomorphometry of femur sample was observed under light microscope.

Statistical Analysis Statistical analysis was performed with GraphPad Prism 7 (GraphPad Software, CA, U.S.A.). Statistical significance was calculated using one-way ANOVA followed by a Tukey's test. All data are presented as the mean \pm standard deviation (S.D.) of at least three independent experiments. $p<0.05$ was considered statistically significant.

\section{RESULTS}

Hsa circ 0002060 Knockdown Ameliorated DEX-Induced Viability Decline of hFOB1.19 Cells In order to mimic OP in vitro, $\operatorname{DEX}(0,0.1,0.5,1,5 \mu \mathrm{M})$ were used to stimulate hFOB1.19 cells. As shown in Fig. 1A, $1 \mu \mathrm{M}$ DEX induced moderate cell viability decrease. Therefore, DEX of $1 \mu \mathrm{M}$ was used in the following experiments in vitro. Next, hFOB1.19 cells were transfected with siRNA 1, siRNA 2, siRNA 3 or NC to knockdown hsa circ 0002060 for $48 \mathrm{~h}$. The results of RT-qPCR demonstrated that siRNA 2 achieved the best knockdown effect (Fig. 1B). Thus, siRNA 2 was applied to knockdown hsa_circ_0002060 in the following experiments. In addition, DEX resulted in the upregulation of hsa_circ_0002060 on a dose dependent manner (Fig. 1C). Meanwhile, DEX-induced upregulation of hsa_circ_0002060
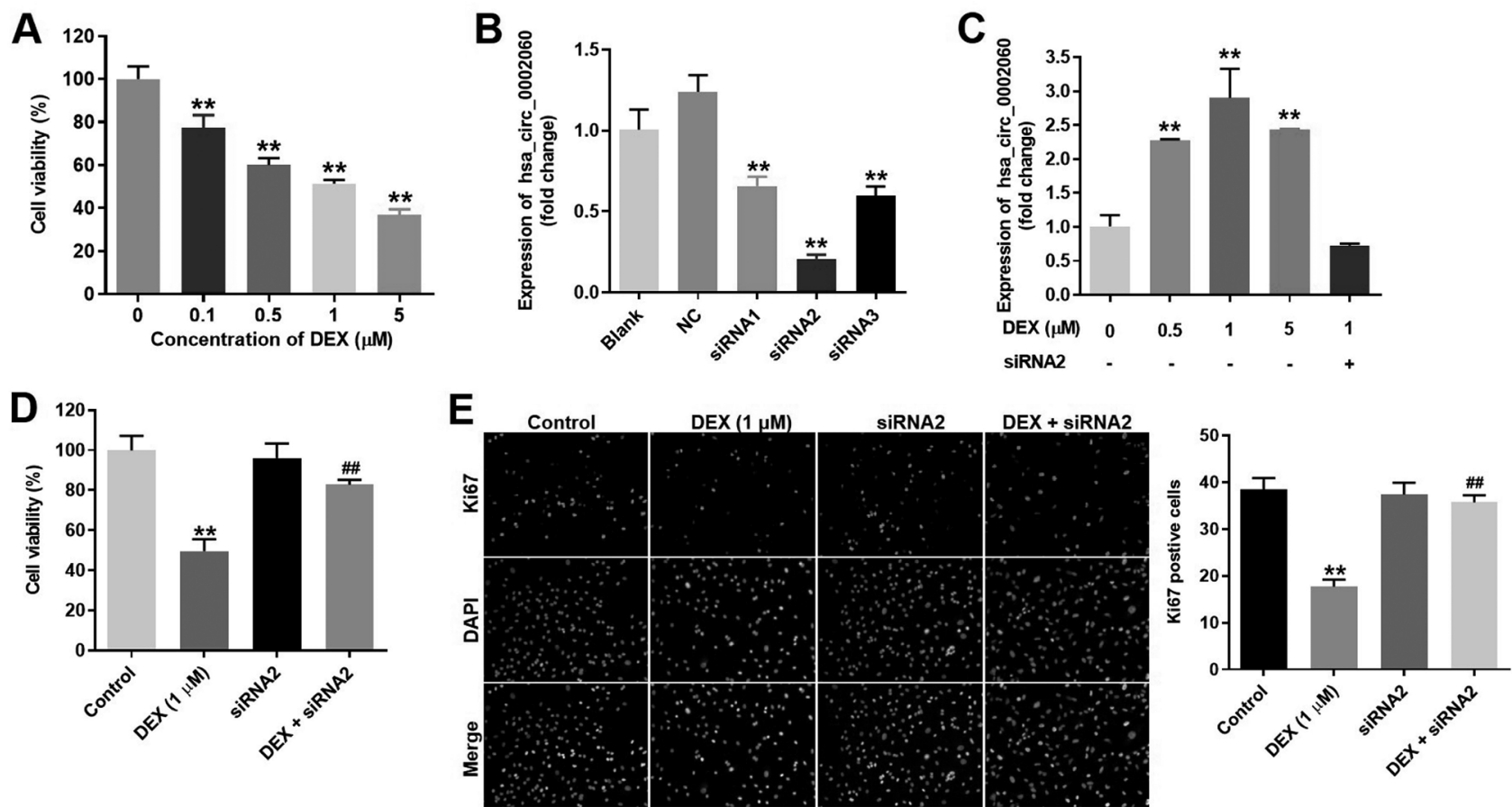

Fig. 1. Hsa_circ_0002060 Knockdown Ameliorated DEX-Induced Viability Decline of hFOB1.19 Cells

(A) The hFOB1.19 cells were treated with DEX $(0,0.1,0.5,1,5 \mu \mathrm{M})$ for $24 \mathrm{~h}$. The effect of DEX on the cell viability was evaluated by CCK-8 assay. (B) hFOB1.19 cells were transfected with siRNA 1 , siRNA 2, siRNA 3 or NC for $48 \mathrm{~h}$. The level of hsa_circ_0002060 was determined by RT-qPCR. (C) hFOB1.19 cells were treated with siRNAs transfection for $48 \mathrm{~h}$, following treated with DEX $(0,0.5,1,5 \mu \mathrm{M})$ for $24 \mathrm{~h}$. RT-qPCR was used to measure the level of hsa circ 0002060 in each group. (D) CCK-8 assay was employed to determine the cell viability. (E) Ki67 staining was utilized to determine the cell proliferation. $* * p<0.01$, compared with control group. $\# p<0.01$, compared with DEX group. $N=3$ per group. 
A

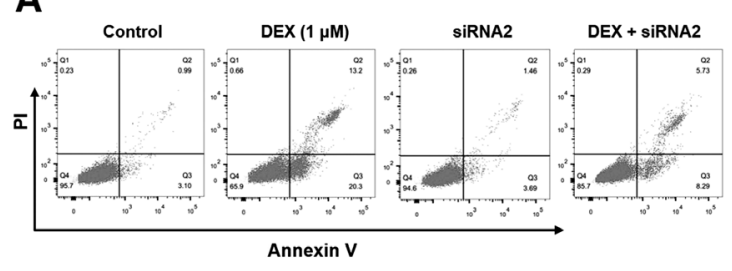

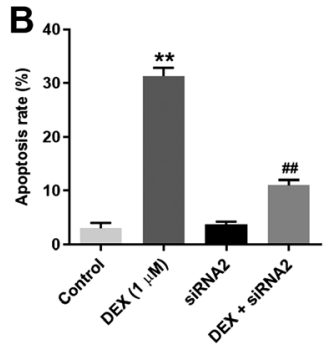

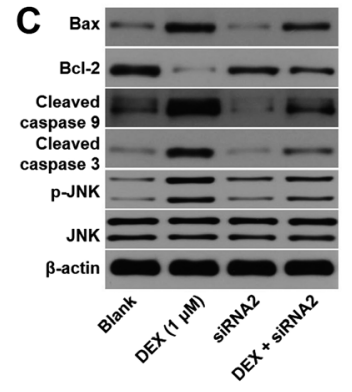

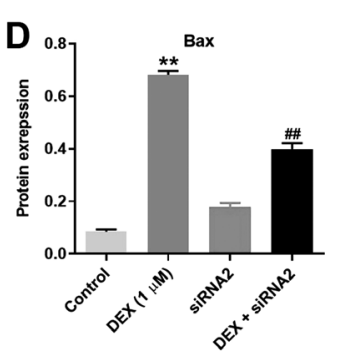
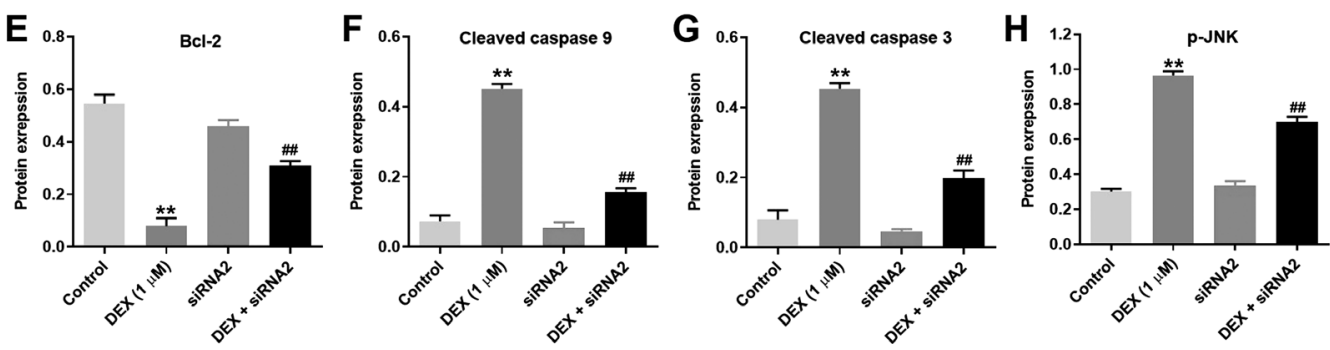

Fig. 2. Hsa_circ_0002060 Knockdown Alleviated DEX-Induced Apoptosis of hFOB1.19 Cells

hFOB1.19 cells were treated with siRNAs transfection for $48 \mathrm{~h}$, following treated with DEX $(0,0.5,1,5 \mu \mathrm{M})$ for $24 \mathrm{~h}$. (A) The cell apoptosis was estimated by Annexin V/PI double staining followed by flow cytometry. (B) The apoptosis rate was quantified. (C) The expression of Bax, bcl-2, cleaved caspase 9 and cleaved caspase 3 were detected using Western blot. (D-G) The levels of Bax, bcl-2, cleaved caspase 9 and cleaved caspase 3 were quantified respectively. $\beta$-Actin was used as inner control for Western blot assay. ${ }^{* *} p<0.01$, compared with control group. ${ }^{* \#} p<0.01$, compared with DEX group. $N=3$ per group.

\section{A}

Hsa_circ_0002060 5'-...-3' CUGUCUGCCACGGAGGUGGACAA (WT)
miR-198-5p 3'-...'5 CUUGGAUAGAGGGGAGACCUGG
Hsa_circ_0002060 5'-...-3' CUGUCUGCCACGGAGGGAAUUAA (MT)

B

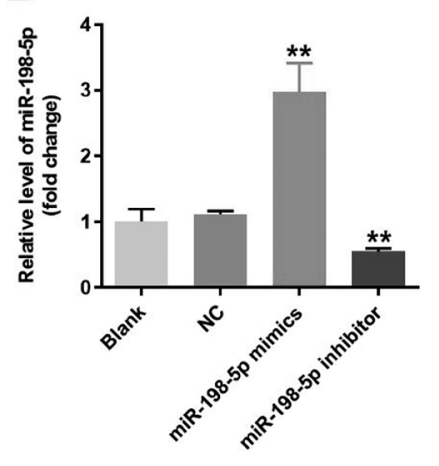

C

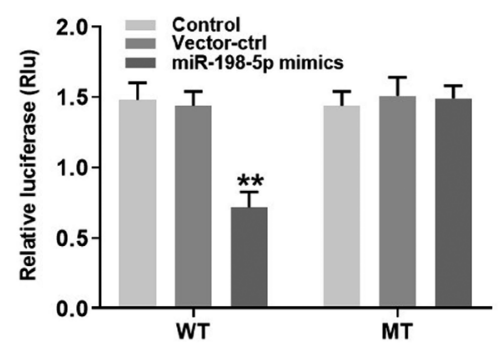

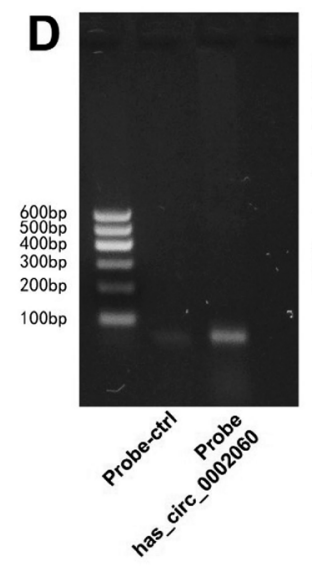
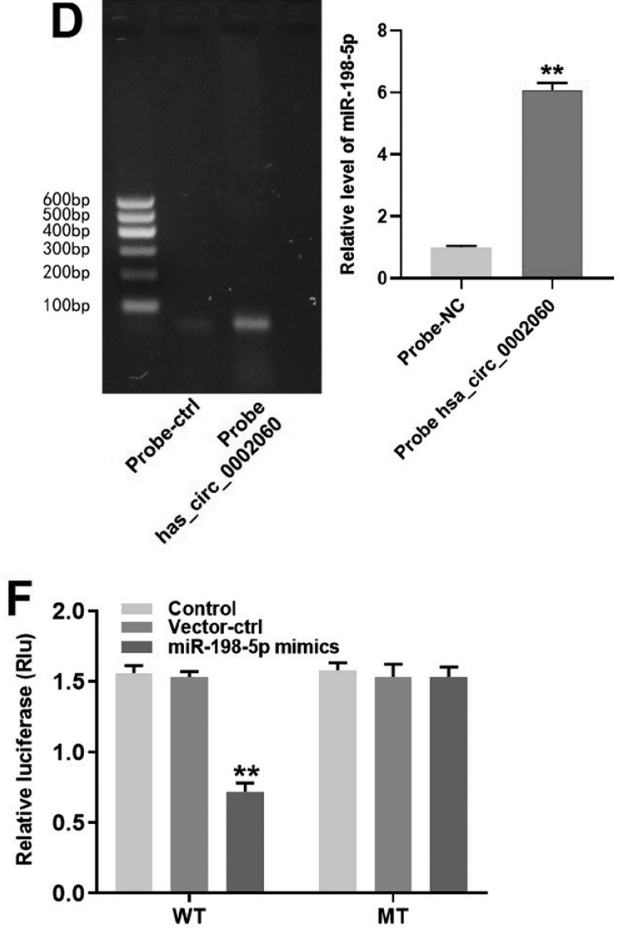

Fig. 3. Hsa_circ_0002060 Targeted MiR-198-5p/Bax Axis

(A) MiR-198-5p was the putative miRNA target of hsa circ 0002060. (B) The hFOB1.19 cells were transfected with miR-198-5p mimics, miR-198-5p inhibitor or NC for 48 $\mathrm{h}$. The efficiency of transfection was determined by measuring level of miR-198-5p using RT-qPCR. (C) The hFOB1.19 cells were co-transfected with WT or MT type of hsa_circ_0002060 and miR-198-5p mimics or vector-ctrl. After $48 \mathrm{~h}$ of transfection, duel luciferase assay was performed. (D) RNA pull down experiment were performed using hsa circ 0002060 probe. Precipitated miR-198-5p was quantified by RT-qPCR. (E) Bax was predicted as the potential target of miR-198-5p. (F) The hFOB1.19 cells were co-transfected with WT or MT type of Bax and miR-198-5p mimics or vector-ctrl. After $48 \mathrm{~h}$ of transfection, duel luciferase assay was performed. The cells without any treatment were used as control. ${ }^{* *} p<0.01$, compared with control group. ${ }^{\# \#} p<0.01$, compared with DEX group. $N=3$ per group. 

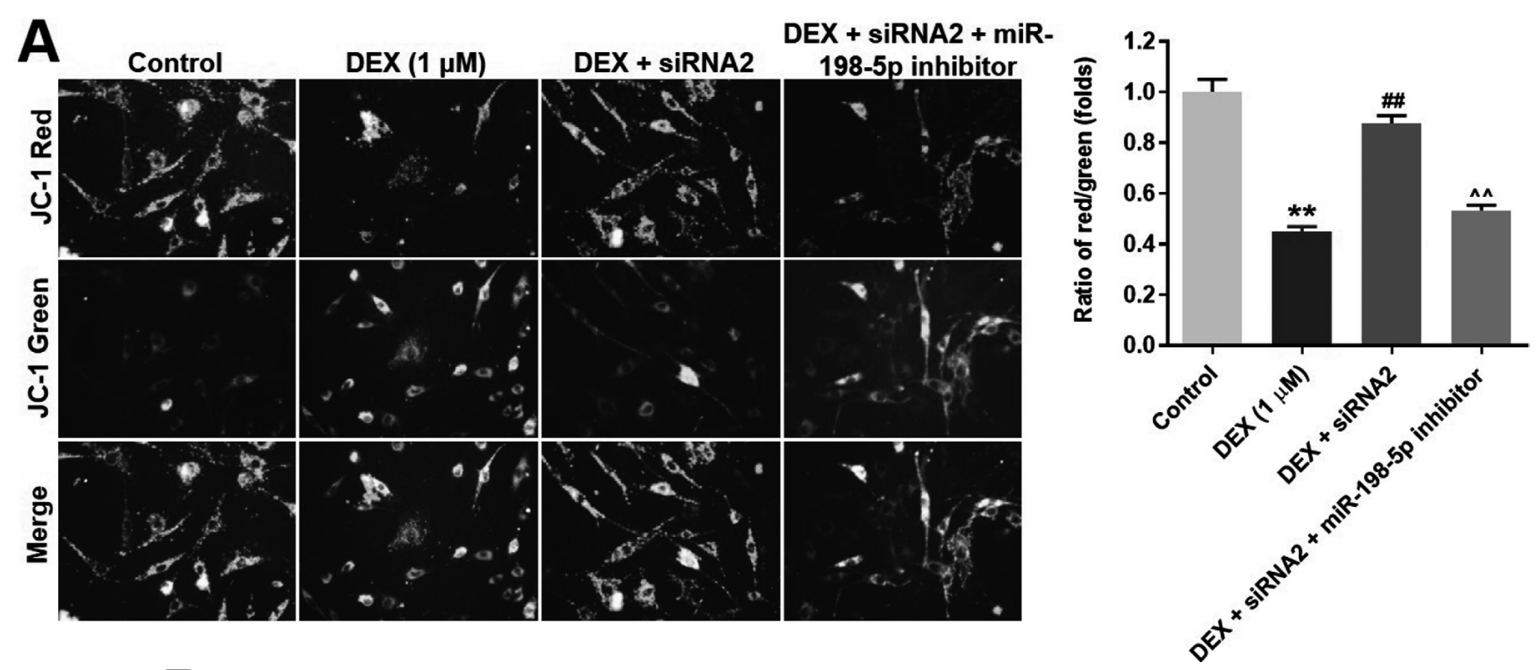

B
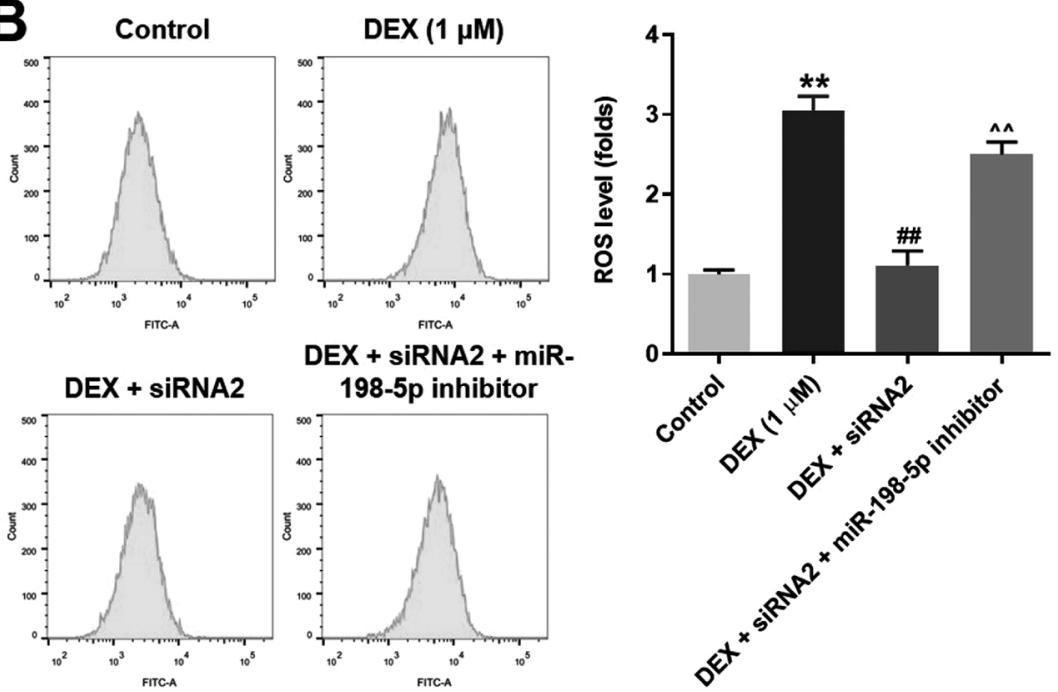

DEX + siRNA2 + miR 198-5p inhibitor

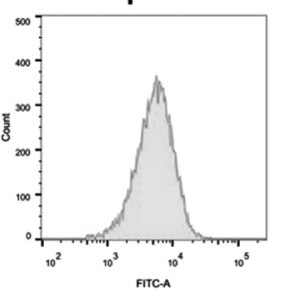

Fig. 4. Hsa_circ_0002060 Knockdown Induced Alterations in MMP and ROS Were Reversed by MiR-198-5p Inhibitor

hFOB1.19 cells were treated with siRNAs transfection for $48 \mathrm{~h}$, following treated with DEX $(0,0.5,1,5 \mu \mathrm{M})$ for $24 \mathrm{~h}$. (A) JC-1 staining was conducted to measure the change of MMP. MMP alterations were quantified as a ratio of red to green fluorescence. (B) After indicated treatment, ROS level in each group was detected and quantified. The cells without any treatment were used as control. ${ }^{* *} p<0.01$, compared with control group. ${ }^{* \#} p<0.01$, compared with DEX group. ${ }^{\wedge} p<0.01$, compared with DEX+ siRNA 2 group. $N=3$ per group.

was significantly inhibited by hsa circ 0002060 siRNA2 (Fig. 1C). Subsequently, the effect of hsa_circ_0002060 knockdown on the cell viability was detected by CCK- 8 assay as well as Ki67 staining. The results indicated that DEX $(1 \mu \mathrm{M})$ lead to viability and proliferation decline in hFOB1.19 cells (Figs. 1D, E). Meanwhile, this phenomenon was reversed by the treatment of hsa_circ_0002060 siRNA 2. Therefore, hsa_circ_0002060 knockdown was able to ameliorate DEXinduced viability decline in hFOB1.19 cells.

Hsa_circ_0002060 Knockdown Alleviated DEX-Induced Apoptosis of hFOB1.19 Cells Next, Annexin V/PI double staining was performed to estimate the effect of hsa_circ_0002060 knockdown on cell apoptosis. The results illustrated that DEX induced significant apoptosis in hFOB1.19 cells (Figs. 2A, B). Moreover, DEX-induced apoptosis was alleviated by hsa_circ_0002060 siRNA2 (Figs. 2A, B). Additionally, Western blot assay was conducted to estimate the alterations in apoptosis-associated markers. As shown in Figs. 2C, D, F, G, the expressions of Bax, cleaved caspase 9 and cleaved caspase 3 were increased by DEX. As shown in Figs. $2 \mathrm{C}$ and $\mathrm{E}$, the expression of bcl-2 was decreased by DEX. In the meantime, these DEX-induced alterations in apoptosis-associated biomarkers were all alleviated by hsa_circ_0002060 knockdown. These results demonstrated that hsa_circ_0002060 knockdown could alleviate DEX-induced apoptosis in hFOB1.19 cells. In addition, DEX-induced upregulation in p-JNK was greatly attenuated by hsa_circ_0002060 knockdown, indicating the involvement of JNK signaling pathway in the role of hsa_circ_0002060 (Figs. 2C, H).

Hsa_circ_0002060 Targeted MiR-198-5p/Bax Axis CircRNAs regulate gene expression by interacting with their target microRNAs as ceRNAs. ${ }^{5)}$ Thus, the miRNA target of hsa_circ_0002060 was predicted using CircInteractome online database. The results of prediction indicated that miR-198-5p was the targeted miRNA of hsa_circ_0002060 (Fig. 3A). Before validating the relationship between hsa_circ_0002060 and miR-198-5p, hFOB1.19 cells were transfected with miR-198-5p mimics, miR-198-5p inhibitor or NC. The level of miR-198-5p was measured by RT-qPCR (Fig. 3B). After $48 \mathrm{~h}$ of transfection, duel luciferase assay was performed to verify the relationship between hsa_circ_0002060 and miR-198-5p. 

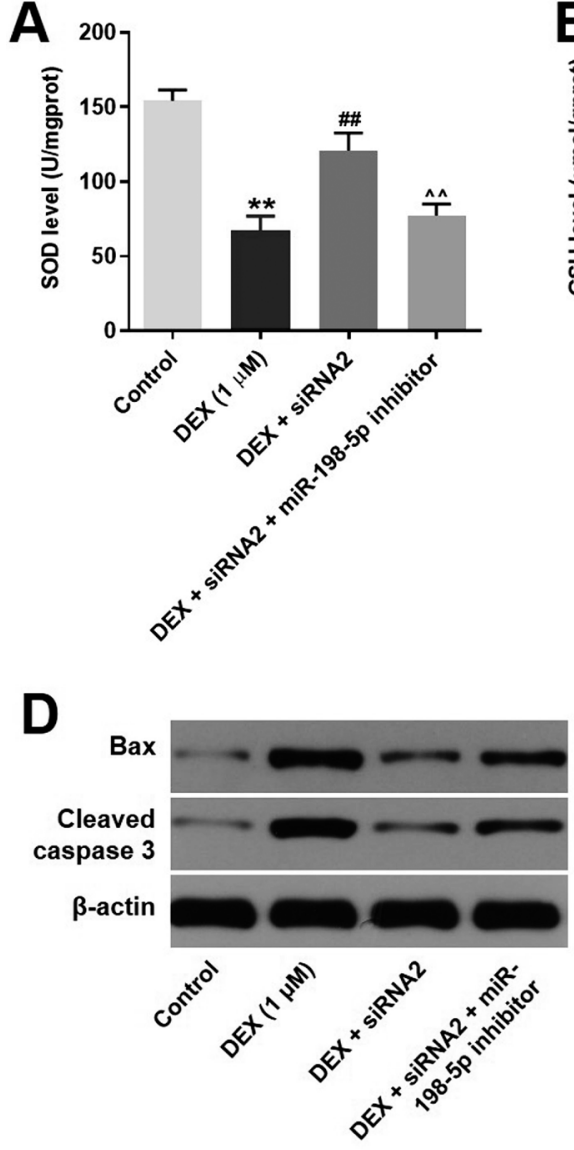
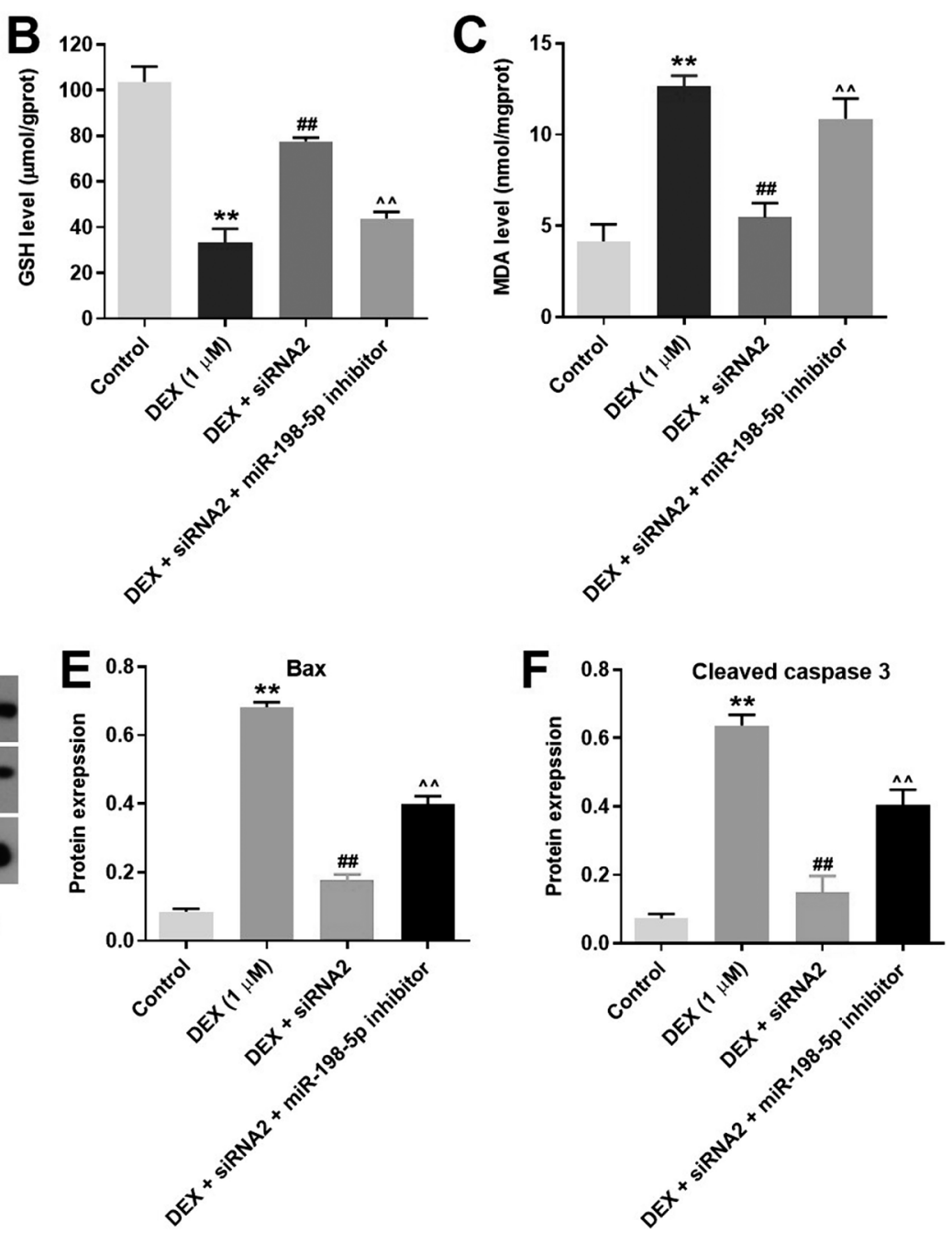

Fig. 5. Hsa_circ_0002060 Knockdown Induced Alterations in Oxidative Stress Indicators and Apoptosis-Associated Biomarkers Were Reversed by MiR-198-5p Inhibitor

hFOB1.19 cells were treated with siRNAs transfection for $48 \mathrm{~h}$, following treated with DEX $(0,0.5,1,5 \mu \mathrm{M})$ for $24 \mathrm{~h}$. (A-C) The levels of SOD, GSH and MDA in each group were detected using ELISA. (D) Western blot assay was performed to detect the expression of Bax and cleaved caspase 3. (E, F) The expressions of Bax and cleaved caspase 3 were quantified. $\beta$-Actin was used as inner control. ${ }^{* *} p<0.01$, compared with control group. ${ }^{\# \#} p<0.01$, compared with DEX group. ${ }^{\wedge} p<0.01$, compared with DEX+ siRNA 2 group. $N=3$ per group.

The results demonstrated that the relative luciferase activity of WT group was significantly reduced compared with control group (Fig. 3C). However, the luciferase activity of MT group was not decreased (Fig. 3C). In addition, RNA pull down assay was conducted to validate the relationship between hsa_circ_0002060 and miR-198-5p. As demonstrated in Fig. 3D, miR-198-5p was precipitated by hsa_circ_0002060 probe. Next, the potential target of miR-198-5p was predicted using TargetScan and miRNDA onlie database. Bax was predicted as the target of miR-198-5p (Fig. 3E). Additionally, the duel luciferase assay proved that WT type of 3'UTR of Bax interacted with miR-198-5p mimics and decreased relatively luciferase activity (Fig. 3F). However, the relatively luciferase activity in MT type group was not declined. All these results illustrated that hsa_circ_0002060 targeted miR-198-5p/Bax axis.

Hsa circ 0002060 Knockdown Induced Alterations in MMP and ROS Were Reversed by MiR-198-5p Inhibitor In addition, JC-1 staining was performed to detect the MMP of hFOB1.19 cells. As shown in Fig. 4A, DEX could cause decreased MMP in hFOB1.19 cells, which was alleviated by hsa circ 0002060 siRNA 2. Furthermore, this phenomenon was reversed in presence of miR-198-5p inhibitor. Consistently, DEX resulted in increased ROS in hFOB1.19 cells, which was inhibited by hsa_circ_0002060 siRNA 2 (Fig. 4B). Moreover, the effect of hsa_circ_0002060 siRNA2 on the change of ROS was reversed in the presence of miR-198-5p inhibitor. According to these results, hsa_circ_0002060 knockdown induced alterations in MMP and ROS were reversed by miR-198-5p inhibitor. These results indicated that hsa_circ_0002060 knockdown protected hFOB1.19 cells from decreased MMP and increased ROS through targeting miR-198-5p.

Hsa_circ_0002060 Knockdown Induced Alterations in Oxidative Stress Indicators and Apoptosis-Associated Biomarkers Were Reversed by MiR-198-5p Inhibitor The levels of SOD, GSH and MDA in hFOB1.19 cells were detected by ELISA kits to evaluate the changes of oxidative stress. As demonstrated in Figs. 5A-C, DEX decreased the level of SOD and GSH but increased the level of MDA in hFOB1.19 cells. Hsa_circ_0002060 siRNA2 inhibited DEXinduced alterations in SOD, GSH and MDA. However, the 

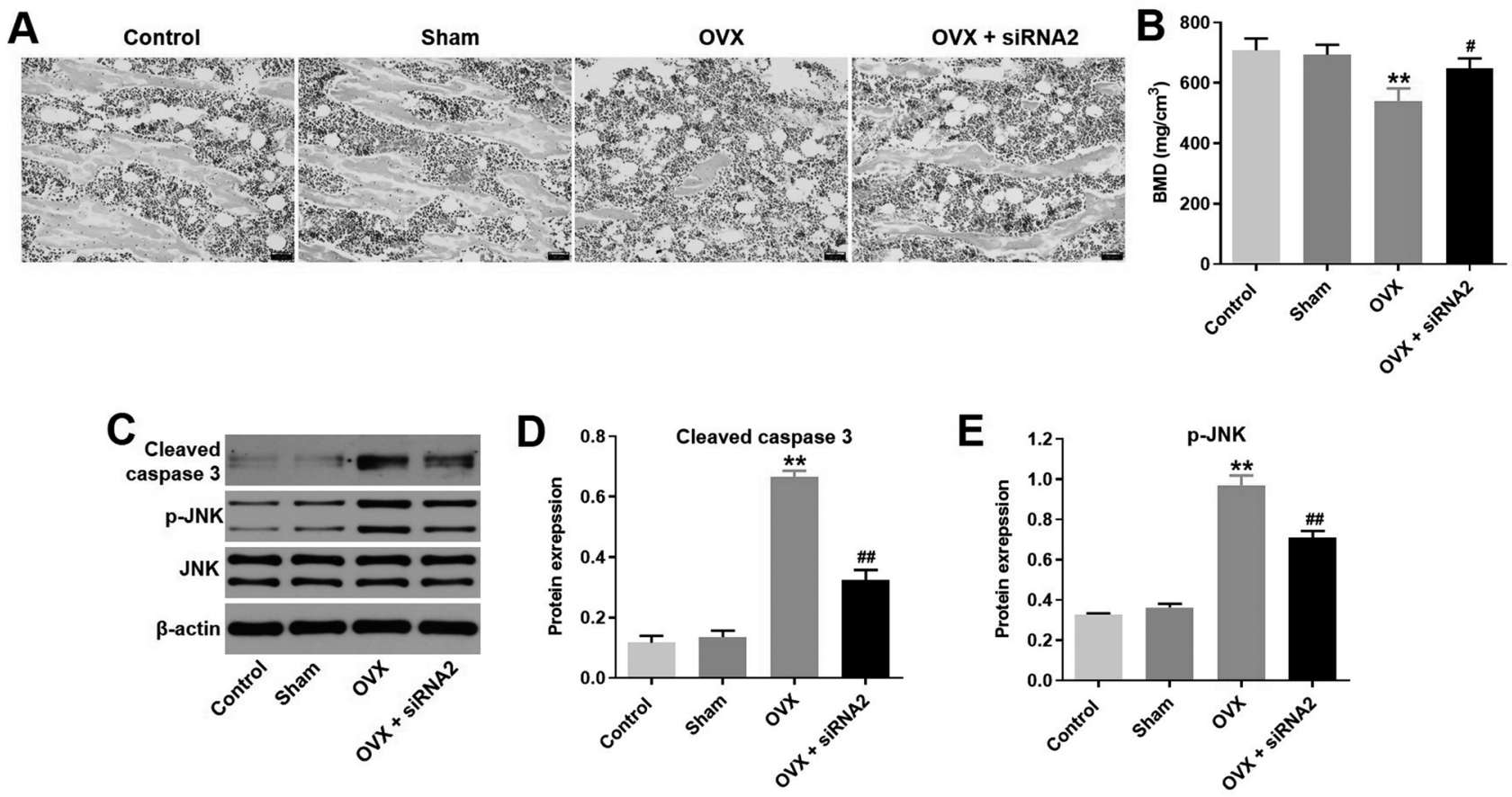

Fig. 6. Hsa_circ_0002060 Knockdown Alleviated Ovariectomy-Induced OP in Vivo

C57BL/6 mice were treated with sham, OVX or OVX + siRNA2 $(50 \mathrm{nM})$. Left femurs were harvested after the mice were sacrificed. (A) H\&E staining of femurs $(N=6)$. (B) Bone mineral density (BMD) was measured. (C-E) Western blot were employed to detect the expression of cleaved caspase 3, p-JNK and JNK in the isolated proteins from the femurs. ${ }^{* *} p<0.01$, compared with control group. ${ }^{\#} p<0.01$, compared with OVX group.

effects of hsa_circ_0002060 siRNA2 on these alterations were reversed in the presence of miR-198-5p inhibitor. Additionally, Western blot was employed to estimate the level of Bax and cleaved caspase 3. The results of Western blot showed hsa_circ_0002060 knockdown could alleviate DEX-induced increase in Bax and cleaved caspase 3. Moreover, the effect of hsa_circ_0002060 knockdown was reversed by miR-198-5p inhibitor. All these results proved that hsa_circ_0002060 knockdown protected hFOB1.19 cells against DEX-induced oxidative stress and apoptosis via targeting miR-198-5p.

Hsa_circ_0002060 Knockdown Alleviated OvariectomyInduced OP in Vivo Finally, the role of hsa_circ_0002060 knockdown in the progression of OP was investigated by using with an OVX-induced OP mouse model. H\&E staining and the measurement of BMD were used to evaluate the effect of hsa_circ_0002060 knockdown in the progression of OP. As indicated in Fig. 6A, OVX resulted in a significant decrease of osteoblast in mouse, while this phenomenon was ameliorated by hsa_circ_0002060 knockdown. In addition, OVX surgery notably decreased the BMD of mouse, which was reversed by hsa_circ_0002060 knockdown as well (Fig. 6B). Moreover, the results of Western blot assay illustrated that OVX induced upregulation of cleaved caspase 3 and p-JNK in femur tissues (Figs. 6C-E). Meanwhile, OVX-induced upregulation of cleaved caspase 3 and p-JNK were all inhibited by hsa_circ_0002060 knockdown (Figs. 6C-E). All these results demonstrated that hsa circ 0002060 knockdown alleviated the progression of OP in vivo through JNK signaling pathway.

\section{DISCUSSION}

At present, the limitations of current therapeutic drugs for OP are including considerable side effects and low efficacy. ${ }^{1)}$ Further research is necessary to explore new thera- peutic target and to develop new drugs for the treatment of OP. One of previous of studies of Huang et al. reported that has_circ_0002060 was upregulated in patients with OP compared with non-OP controls using bioinformatics analysis. ${ }^{6}$ Meanwhile, has_circ_0002060 was found to be associated with low bone mineral density, revealing that has_circ_0002060 could be used as a therapeutic target or a potential diagnostic biomarker. ${ }^{6)}$ However, further research on has_circ_0002060 in vitro and in vivo is still lacking. The mechanisms underlying the regulation effect of has_circ_0002060 also remain to be explored. Our findings for the first time, proved the regulation effect of has_circ_0002060 in the viability of hFOB1.19 cells, which was in line with the findings of Huang et al. In addition, the findings of the present study demonstrated that hsa_circ_0002060 targeted miR-198-5p/Bax axis, by which has_circ_0002060 acted its role in hFOB1.19 cells. These findings of mechanisms are valuable to understand the pathology of OP, as well as helpful to develop new therapeutic drugs. Moreover, our present study proved the effect of has_circ_0002060 knockdown on alleviating OP in an OVX mouse model. These findings from in vivo experiments shed light on the potentials of has_circ_0002060 as a novel therapeutic target for OP.

Previous studies already proved that the activity of Bcl-2 is mediated by the upstream protein kinase JNK. ${ }^{7)}$ In this study, has_circ_0002060 knockdown remarkably reversed DEX-induced downregulation of Bcl-2 level. Meanwhile, DEX-induced upregulation of p-JNK was inhibited by has_circ_0002060 knockdown. Taken these results together, hsa_circ_0002060 might regulate Bcl-2 through JNK signaling pathway, which is in line with previous studies. In addition, it was previously reported that the activation of JNK played a key role during oxidative stress induced apoptosis. ${ }^{8)}$ Moreover, Zhu et al. reported that betulinic acid protected 
mice from DEX-induced oxidative stress through mediating JNK involved signaling pathway. DEX-induced oxidative damage was alleviated by increasing total antioxidant capacity. ${ }^{9)}$ The activity of SOD was increased and the MDA level was decreased by betulinic acid through JNK signaling pathway. ${ }^{9)}$ Consistently, we found that $\mathrm{p}-\mathrm{JNK}$ was activated by DEX in hFOB 1.19 cells and upregulated by OVX treatment in mice. Meanwhile, DEX-induced and OVX-induced activation of p-JNK were all alleviated by hsa circ 0002060 knockdown. Furthermore, hsa_circ_0002060 knockdown increased the levels of SOD and GSH and decreased the level of MDA in hFOB 1.19 cells. Thus, we deduce that the protective effect of hsa_circ_0002060 knockdown in the progression of OP was also mediated by JNK signaling pathway.

In addition, the involvement of JNK signaling pathway in the growth of osteoblast was also revealed in other previous studies. Guo et al. reported that lipopolysaccharide (LPS) significantly elevated the protein expression of Bax and caspase-3, whereas downregulated bcl-2 in MC3T3-E1 cells through promoting the phosphorylation of $\mathrm{JNK}^{10)}$ Our findings of current further validated the involvement of JNK signaling pathway in the progression of OP. However, more detailed regulatory mechanisms were still waiting to be explored. The relationship between miR-198-5p/Bax axis and JNK signaling pathway in the role of hsa_circ_0002060 on regulating OP was unrevealed yet.

\section{CONCLUSION}

Hsa_circ_0002060 knockdown could protect hFOB 1.19 cells from $\bar{D} E X$-induced cell viability decline through targeting miR-198-5p. Hsa_circ_0002060 might possibly be regarded as a novel therapeutic target for the treatment of OP.

Acknowledgments This study was supported by the funding of Key Basic Research Project of Shenzhen Polytechnic (\#6019310002 K) and Project of Science and Technology Plan of Shenzhen (\#JCYJ20180228164957104).
Conflict of Interest The authors declare no conflict of interest.

Supplementary Materials The online version of this article contains supplementary materials.

\section{REFERENCES}

1) Chen LR, Ko NY, Chen KH. Medical treatment for osteoporosis: from molecular to clinical opinions. Int. J. Mol. Sci., 20, 2213 (2019).

2) Sözen T, Özışık L, Başaran NC. An overview and management of osteoporosis. Eur. J. Rheumatol., 4, 46-56 (2017).

3) Hua X, Sun Y, Chen J, Wu Y, Sha J, Han S, Zhu X. Circular RNAs in drug resistant tumors. Biomed. Pharmacother., 118, 109233 (2019).

4) Gomes CPC, Salgado-Somoza A, Creemers EE, Dieterich C, Lustrek M, Devaux Y. Circular RNAs in the cardiovascular system. Noncoding RNA Res., 3, 1-11 (2018).

5) Jiang WD, Yuan PC. Molecular network-based identification of competing endogenous RNAs in bladder cancer. PLOS ONE, 14, e0220118 (2019).

6) Huang Y, Xie J, Li E. Comprehensive circular RNA profiling reveals circ 0002060 as a potential diagnostic biomarkers for osteoporosis. J. Cell. Biochem., 120, 15688-15694 (2019).

7) Fan J, Liu Y, Yin J, Li Q, Li Y, Gu J, Cai W, Yin G. Oxygenglucose-deprivation/reoxygenation-induced autophagic cell death depends on JNK-mediated phosphorylation of Bcl-2. Cell. Physiol. Biochem., 38, 1063-1074 (2016).

8) Liu S, Shen M, Li C, Wei Y, Meng X, Li R, Cao Y, Wu W, Liu H. $\mathrm{PKC} \delta$ contributes to oxidative stress-induced apoptosis in porcine ovarian granulosa cells via activating JNK. Theriogenology, 131, 89-95 (2019)

9) Zhu L, Yi X, Zhao J, Yuan Z, Wen L, Pozniak B, ObminskaMrukowicz B, Tian Y, Tan Z, Wu J, Yi J. Betulinic acid attenuates dexamethasone-induced oxidative damage through the JNK-P38 MAPK signaling pathway in mice. Biomed. Pharmacother., 103, 499-508 (2018)

10) Guo C, Yuan L, Wang JG, Wang F, Yang XK, Zhang FH, Song JL, Ma XY, Cheng Q, Song GH. Lipopolysaccharide (LPS) induces the apoptosis and inhibits osteoblast differentiation through JNK pathway in MC3T3-E1 cells. Inflammation, 37, 621-631 (2014). 